\title{
Physicians' knowledge on specific rare diseases and its associated factors: a national cross-sectional study from China
}

\author{
Huanyu Zhang ${ }^{1 \dagger}$, Ying $\mathrm{Xiao}^{2 \dagger}$, Xinyue Zhao ${ }^{2}$, Zhuang Tiann ${ }^{2}$, Shu-yang Zhang ${ }^{2^{*}+}$ (1) and Dong Dong ${ }^{1,3^{*+}}$
}

\begin{abstract}
Background: Rare disease patients often experience diagnosis delays or misdiagnosis, which may be due to lack of knowledge on rare diseases among physicians.

Objective: To assess Chinese physicians' knowledge on specific rare diseases and identify its associated factors.

Methods: Thirty-four patient organizations with a unique disease of interest were invited to develop 3 knowledge questions for each rare disease to assess physicians' knowledge on the disease that they felt most experienced in. The total knowledge score for each participant ranged from a score of 0 to 3. A national cross-sectional study conducted in a cohort of 3197 physicians from 6 provinces across western, central and eastern China. The demographic information of the participants was collected including gender, age, birthplace, income, education, hospital class, working title, working years, and specialty. A multiple linear regression analysis was performed to assess the independent associations between the physician variables and the total knowledge score.
\end{abstract}

Results: Two thousand, one hundred and fifteen (66.16\%) of the involved physicians obtained a total knowledge score of 2 or 3 . The median knowledge scores of $10(29.4 \%)$ rare diseases were a score of 1.5 or below. Physicians with female gender ( $\beta=0.08, p<0.05$ for females vs. males), and a monthly income of 5000-10,000 RMB ( $\beta=0.11, p<0.01$ for $5000-10,000$ vs. $<5000)$ and 10,000-30,000 RMB $(\beta=0.14, p<0.05)$ were associated with a higher score. Specialties of physicians who received a relatively higher score included internal medicine, obstetrics and gynecology, radiology, intensive care unit, and surgery.

Conclusions: Almost two thirds of the participants had an average or good level of knowledge on the specific rare disease that they felt most experienced in. Physicians with female gender, a monthly income of 5000-10,000 RMB and 10,000-30000 RMB, and specialties of internal medicine, obstetrics and gynecology, radiology, intensive care unit, and surgery, were associated with a relatively higher knowledge score.

Keywords: Physicians, Rare diseases, Knowledge, China, Patient organizations

*Correspondence: shuyangzhang103@nrdrs.org; dongdong@cuhk.edu.hk

${ }^{\dagger}$ Co-first authors: Huanyu Zhang and Ying Xiao

${ }^{\dagger}$ Co-corresponding authors: Shu-yang Zhang and Dong Dong

${ }^{2}$ Department of Cardiology, Peking Union Medical College Hospital,

Chinese Academy of Medical Sciences and Peking Union Medical College, Beijing 100730, China

3 JC School of Public Health and Primary Care, Faculty of Medicine, The Chinese University of Hong Kong, Shatin, Hong Kong SAR, China

Full list of author information is available at the end of the article

\section{Background}

Rare diseases are generally defined as serious, chronic, and often life-threatening conditions which affect a small percentage of population [1]. Among the 6000-8000 rare diseases that have been identified worldwide, $6-8 \%$ of the global population is affected [2,3]. Although each rare disease represents varied problems experienced by patients, families, and caregivers, one of the most 
common issues that prevents patients from achieving a better quality of life is difficulty in diagnosis [4-6]. A survey published in 2013 revealed that it took an average of 7.6 years in the United States and 5.6 years in the United Kingdom for rare disease patients to receive an appropriate diagnosis [7]. Misdiagnosis or diagnosis delays can lead to the deterioration of symptoms and disease progression to rare disease patients, resulting in inappropriate medical interventions and additional medical costs $[4,5,8]$. This situation is not only frustrating to patients and health care professionals, but also challenging to the healthcare systems around the world.

In a traditional patient-physician relationship, physicians often dominate in the medical encounters assuming they have sufficient (and superior) knowledge and skills regarding the diagnosis and treatment. However, in the context of rare diseases, due to low prevalence and high clinical complexity, physicians often lack knowledge and experience, resulting in a shift in the traditional patientphysician interaction [9]. Rare disease patients and their families have to become knowledgeable about their own diseases and educate physicians about their conditions $[4,5,9]$. This role discrepancy may cause further problems. A number of studies have shown that lack of rare disease awareness and knowledge among physicians could lead to delayed diagnosis or misdiagnosis [4, 5, 9-11]. A previous study indicated that $78.8 \%$ of patients affected by rare diseases have not received proper care because of limited training of health care professionals in this field [12].

In China, no epidemiological data on the prevalence of rare diseases is available so far. A recent study conducted in Hong Kong showed that the prevalence of rare diseases was estimated at 1 in 67 in Hong Kong population [13]. Hence, we estimated that approximately 21 million people are affected by rare diseases in China; this number represents a high probability of encountering such patients for health care professionals during their career. Efforts have been made to improve the quality of care for rare disease patients in China since 1980s [14]. In 2018, the first official list of rare diseases in China has been released [15]. Subsequently, a guideline for the diagnosis and treatment of 121 rare diseases defined by the list has been initiated to support clinical practice at the national level. However, difficulty in diagnosis among rare disease patients can still be observed in China. A recent national survey reported that $73 \%$ of the investigated rare disease patients were misdiagnosed; they had to spend an average of 4.3 years and visit 3 hospitals to access the correct diagnosis [6]. Additionally, due to the uneven distribution of healthcare resources in China, rare disease patients and their families often have to travel across provinces to obtain an accurate diagnosis, which could increase financial burden on the family $[6,16]$. Although a few studies conducted in China have highlighted the importance of improving rare disease awareness and knowledge among physicians $[6,15]$, no disease-specific information about these unmet needs is available in China to the best of our knowledge. Hence, this study aimed to assess physicians' knowledge on specific rare diseases and identify its associated factors at the national level in China.

\section{Methods}

\section{Development of knowledge questions on rare diseases}

In this study, we invited patient organizations with a specific disease of interest in China to develop questions so as to assess the degree of physicians' knowledge on rare diseases. The following were the inclusion criteria for patient organizations: (1) the disease of interest of patient organizations is included in the official list of rare diseases in China; (2) an organizer is responsible for routine practice performed in the office or online chat groups regardless of official registration; (3) the patient organization with the largest number of patients or longest history will be selected if several patient organizations with the same disease of interest exist. After applying the inclusion criteria, we invited 50 patient organizations to participate in our study. Consequently, 34 patient organizations with a unique disease of interest agreed to be involved.

The process for developing knowledge questions on rare diseases started from May 2019. The involved patient organizations were required to provide three true-false questions regarding the knowledge on the disease of their interest, with the degree of difficulty ranging from low, medium, to high. A self-assessment on the quality of questions was conducted by the specialists acquainted by the patient organizations. After reviewing the contents and answers of the knowledge questions by researchers in this study, a total of 102 questions on 34 rare diseases were ready for further actions in December 2019 (Additional file 1).

\section{Study sample and data collection}

A cross-sectional study was conducted in January 2020. Physicians from 6 provinces across eastern, central, and western China including Shandong, Jiangsu, Shanxi, Jiangxi, Sichuan, and Shannxi, were investigated. A non-random convenience sampling method was used to recruit physicians aged 18 years or above from around 200 hospitals located in the six provinces. Medical students who had not graduated were not included in the current study. The demographic information of the participants was collected including gender, age, birthplace, income, education, hospital class, working title, working years, and specialty. All the participants were 
anonymized and de-identified; any sensitive information on physicians was not accessible.

Regarding the medical encounter of rare diseases, the participants were investigated if they had experience of encountering a patient with any of 34 rare diseases of concern. Among the 4387 physicians who agreed to participate in the study, $1062(24.2 \%)$ reported that they had no medical encounters of rare disease patients and 128 (2.9\%) reported to have experience of encountering rare disease patients beyond the specific 34 rare diseases of interest; these physicians were excluded from the current study. Eligible participants were further asked to select one rare disease that they felt most experienced in and subsequently answer three knowledge questions regarding this rare disease. The knowledge questions were provided with three options, namely, true, false, and don't know. If the participants didn't choose the correct answer or choose 'don't know' for each question, they would obtain a score of 0 . Otherwise, they would obtain a score of 1 . The total knowledge score for each participant ranged from a score of 0 to 3 .

\section{Statistical analysis}

Frequencies and percentages were calculated for descriptive data based on geographical locations. The median score with interquartile range (IQR) for each specific rare disease was reported. The characteristics of physicians with a total knowledge score ranging from a score of 0 to 3 as a categorical variable were compared. Fisher's exact tests were used to determine the differences between the four groups of physicians. Univariable analyses were conducted to evaluate the relationship between the characteristics of physicians and the total knowledge score as a continuous variable. To assess the independent associations between the physician variables and the total knowledge score, a multiple linear regression analysis was performed. The variance inflation factor (VIF) for each independent variable in the regression model was detected to eliminate multicollinearity. A $p$-value lower than 0.05 was considered statistically significant. All the statistical analyses were conducted using $\mathrm{R}$ version 4.0.4.

\section{Results}

Of the 4387 physicians who agreed to participate in this study, 3197 (72.9\%) had experience of encountering patients with 34 rare diseases of concern. Among them, $54.5 \%$ were females, $44.5 \%$ aged $26-35$ years, and $64.7 \%$ reported a master or higher degree. More detailed information on the demographics of the study sample is presented in Table 1.

In Table 2, medians of the total knowledge score with IQRs for each rare disease were reported. Among the 34 rare diseases of concern, the most frequently chosen rare diseases that physicians felt experienced in were Albinism (14.58\%), Hepatolenticular degeneration (Wilson's Disease) (11.85\%), Multiple Sclerosis (10.85\%), and Hemophilia (9.16\%). The median knowledge scores of 10 (29.4\%) rare diseases were a score of 1.5 or below, including Tetrahydrobiopterin deficiency $(1.00 \pm 0.00)$, Spinal and bulbar muscular atrophy $(1.00 \pm 0.25)$, Type II glycogen storage disease (1.00 \pm 0.50$)$, Amyotrophic lateral sclerosis $(1.00 \pm 1.00)$, Osteogenesis imperfecta $(1.00 \pm$ $1.00)$, Prader-Willi syndrome (1.00 \pm 1.00$)$, Phenylketonuria $(1.00 \pm 1.00)$, Multiple sclerosis $(1.00 \pm 1.00)$, Spinocerebellar ataxia $(1.00 \pm 1.50)$, and Spinal muscular atrophy $(1.50 \pm 1.00)$.

Table 3 compares the characteristics of physicians with a total knowledge score ranging from 0 to 3 as a categorical variable. In this study, 1261 (39.44\%) of the involved physicians obtained a total knowledge score of 2, followed by $858(26.84 \%)$ with a score of $1,854(26.71 \%)$ with a score of 3 , and 224 (7.01\%) with a score of 0 . Significant differences between different groups of physicians were found in gender, birthplace, monthly income, province, education, hospital class, and specialty. Factors associated with the total knowledge score among physicians were identified in Table 4 . The variables that were not significantly associated with the total knowledge score in the univariable analysis were not included in the multiple linear regression analysis. In the multiple linear regression analysis, females were found to acquire a higher knowledge score than males $(\beta=0.08, p<0.05)$. Compared to physicians with a monthly income of below $5000 \mathrm{RMB}$, those with an income of 5000-10,000 RMB $(\beta=0.11, p<0.01)$ and $10,000-30,000$ RMB $(\beta=0.14$, $p<0.05)$ were at a higher probability of obtaining a higher score. Physicians who have worked longer than 30 years were less likely to receive a higher knowledge score than those with working years of 5 or below $(\beta=-0.18$, $p<0.05)$. In contrast to the specialty of internal medicine, specialties of physicians who received a relatively higher score included obstetrics and gynecology $(\beta=-0.15$, $p<0.05)$, radiology $(\beta=-0.16, \quad p<0.05), \quad$ intensive care unit $(\beta=-0.20, p<0.05)$ and surgery $(\beta=-0.24$, $p<0.001)$. All the variables in the reported model were found to have a value of VIF below 5 , which indicated no problem of collinearity.

\section{Discussion}

This study assessed the level of knowledge on rare diseases among Chinese physicians who had medical encounters of 34 rare diseases of concern. Approximately $73 \%$ of the invited participants were eligible for answering knowledge questions provided by 34 patient organizations with a specific disease of interest. This may indicate that most physicians will encounter the diagnosis 
Table 1 Demographic characteristics of physicians who had experience of encountering a patient with any of 34 rare diseases of concern

\begin{tabular}{|c|c|c|c|c|}
\hline \multirow[t]{2}{*}{ Characteristics } & $\begin{array}{l}\text { Total } \\
n=3197\end{array}$ & $\begin{array}{l}\text { Eastern } \\
n=1493\end{array}$ & $\begin{array}{l}\text { Central } \\
n=1427\end{array}$ & $\begin{array}{l}\text { Western } \\
\mathbf{n}=\mathbf{2 7 7}\end{array}$ \\
\hline & \multicolumn{4}{|c|}{ Number (percent) } \\
\hline \multicolumn{5}{|l|}{ Gender } \\
\hline Male & $1456(45.5 \%)$ & $724(48.5 \%)$ & $620(43.4 \%)$ & $112(40.4 \%)$ \\
\hline Female & $1741(54.5 \%)$ & 769 (51.5\%) & 807 (56.6\%) & $165(59.6 \%)$ \\
\hline \multicolumn{5}{|l|}{ Age } \\
\hline $18-25$ & $41(1.3 \%)$ & $14(0.9 \%)$ & $22(1.5 \%)$ & $5(1.8 \%)$ \\
\hline $26-35$ & $1424(44.5 \%)$ & $695(46.6 \%)$ & $588(41.2 \%)$ & $141(50.9 \%)$ \\
\hline $36-45$ & 1075 (33.6\%) & $516(34.6 \%)$ & $484(33.9 \%)$ & 75 (27.1\%) \\
\hline $46-55$ & $544(17.0 \%)$ & $223(14.9 \%)$ & $276(19.3 \%)$ & $45(16.2 \%)$ \\
\hline $56-65$ & $106(3.3 \%)$ & $43(2.9 \%)$ & $53(3.7 \%)$ & $10(3.6 \%)$ \\
\hline $66+$ & $7(0.2 \%)$ & $2(0.1 \%)$ & $4(0.3 \%)$ & $1(0.4 \%)$ \\
\hline \multicolumn{5}{|l|}{ Birthplace } \\
\hline Urban area & 2843 (88.9\%) & $1321(88.5 \%)$ & 1285 (90.0\%) & $237(85.6 \%)$ \\
\hline Rural area & $351(11.0 \%)$ & $171(11.5 \%)$ & $140(9.8 \%)$ & $40(14.4 \%)$ \\
\hline Others & $3(0.1 \%)$ & $1(0.1 \%)$ & $2(0.1 \%)$ & $0(0.0 \%)$ \\
\hline \multicolumn{5}{|l|}{ Monthly income } \\
\hline$<5000$ & $653(20.4 \%)$ & $118(7.9 \%)$ & $494(34.6 \%)$ & $41(14.8 \%)$ \\
\hline $5000-10,000$ & 2026 (63.4\%) & $995(66.6 \%)$ & $855(59.9 \%)$ & $176(63.5 \%)$ \\
\hline $10,000-30,000$ & $506(15.8 \%)$ & $376(25.2 \%)$ & $71(5.0 \%)$ & $59(21.3 \%)$ \\
\hline $30,000-50,000$ & $7(0.2 \%)$ & $2(0.1 \%)$ & $5(0.4 \%)$ & $0(0.0 \%)$ \\
\hline$>50,000$ & $5(0.2 \%)$ & $2(0.1 \%)$ & $2(0.1 \%)$ & $1(0.4 \%)$ \\
\hline \multicolumn{5}{|l|}{ Province } \\
\hline Shandong & $298(9.3 \%)$ & $298(20.0 \%)$ & $0(0.0 \%)$ & $0(0.0 \%)$ \\
\hline Jiangsu & 1195 (37.4\%) & 1195 (80.0\%) & $0(0.0 \%)$ & $0(0.0 \%)$ \\
\hline Jiangxi & $468(14.6 \%)$ & $0(0.0 \%)$ & $468(32.8 \%)$ & $0(0.0 \%)$ \\
\hline Shanxi & $959(30.0 \%)$ & $0(0.0 \%)$ & $959(67.2 \%)$ & $0(0.0 \%)$ \\
\hline Sichuan & $178(5.6 \%)$ & $0(0.0 \%)$ & $0(0.0 \%)$ & $178(64.3 \%)$ \\
\hline Shannxi & $99(3.1 \%)$ & $0(0.0 \%)$ & $0(0.0 \%)$ & $99(35.7 \%)$ \\
\hline \multicolumn{5}{|l|}{ Education } \\
\hline Secondary vocational school & $3(0.1 \%)$ & $0(0.0 \%)$ & $2(0.1 \%)$ & $1(0.4 \%)$ \\
\hline Three-year college & $19(0.6 \%)$ & $3(0.2 \%)$ & $10(0.7 \%)$ & $6(2.2 \%)$ \\
\hline Bachelor's degree & $1104(34.5 \%)$ & 345 (23.1\%) & $667(46.7 \%)$ & $92(33.2 \%)$ \\
\hline Master's degree & $1782(55.7 \%)$ & $927(62.1 \%)$ & 727 (50.9\%) & $128(46.2 \%)$ \\
\hline Doctorate/postdoc & $289(9.0 \%)$ & $218(14.6 \%)$ & $21(1.5 \%)$ & $50(18.1 \%)$ \\
\hline \multicolumn{5}{|l|}{ Title } \\
\hline Primary & $823(25.7 \%)$ & $334(22.4 \%)$ & $384(26.9 \%)$ & $105(37.9 \%)$ \\
\hline Middle & $1208(37.8 \%)$ & $584(39.1 \%)$ & $544(38.1 \%)$ & $80(28.9 \%)$ \\
\hline Vice-senior & $709(22.2 \%)$ & $350(23.4 \%)$ & $298(20.9 \%)$ & $61(22.0 \%)$ \\
\hline Senior & $435(13.6 \%)$ & $217(14.5 \%)$ & $190(13.3 \%)$ & $28(10.1 \%)$ \\
\hline None & $22(0.7 \%)$ & $8(0.5 \%)$ & $11(0.8 \%)$ & $3(1.1 \%)$ \\
\hline \multicolumn{5}{|l|}{ Working years } \\
\hline$\leq 5$ & $828(25.9 \%)$ & $396(26.5 \%)$ & $348(24.4 \%)$ & 84 (30.3\%) \\
\hline $6-10$ & $779(24.4 \%)$ & 376 (25.2\%) & $327(22.9 \%)$ & $76(27.4 \%)$ \\
\hline $11-15$ & $514(16.1 \%)$ & $217(14.5 \%)$ & $254(17.8 \%)$ & $43(15.5 \%)$ \\
\hline $16-20$ & $368(11.5 \%)$ & 194 (13.0\%) & $156(10.9 \%)$ & $18(6.5 \%)$ \\
\hline $21-25$ & $333(10.4 \%)$ & 154 (10.3\%) & $154(10.8 \%)$ & $25(9.0 \%)$ \\
\hline $26-30$ & $216(6.8 \%)$ & 92 (6.2\%) & 105 (7.4\%) & $19(6.9 \%)$ \\
\hline $30+$ & $159(5.0 \%)$ & $64(4.3 \%)$ & $83(5.8 \%)$ & $12(4.3 \%)$ \\
\hline
\end{tabular}


Table 1 (continued)

\begin{tabular}{|c|c|c|c|c|}
\hline \multirow[t]{2}{*}{ Characteristics } & $\begin{array}{l}\text { Total } \\
n=3197\end{array}$ & $\begin{array}{l}\text { Eastern } \\
n=1493\end{array}$ & $\begin{array}{l}\text { Central } \\
n=1427\end{array}$ & $\begin{array}{l}\text { Western } \\
\mathrm{n}=\mathbf{2 7 7}\end{array}$ \\
\hline & \multicolumn{4}{|c|}{ Number (percent) } \\
\hline \multicolumn{5}{|l|}{ Hospital class } \\
\hline Tertiary hospital & $3185(99.6 \%)$ & 1492 (99.9\%) & 1425 (99.9\%) & $268(96.8 \%)$ \\
\hline Secondary hospital & $8(0.3 \%)$ & $1(0.1 \%)$ & $0(0.0 \%)$ & $7(2.5 \%)$ \\
\hline Primary hospital & $4(0.1 \%)$ & $0(0.0 \%)$ & $2(0.1 \%)$ & $2(0.7 \%)$ \\
\hline \multicolumn{5}{|l|}{ Specialty } \\
\hline Anesthesiology & $33(1.0 \%)$ & $6(0.4 \%)$ & $27(1.9 \%)$ & $0(0.0 \%)$ \\
\hline Dermatology & $57(1.8 \%)$ & $21(1.4 \%)$ & $32(2.2 \%)$ & $4(1.4 \%)$ \\
\hline Emergency medicine & $89(2.8 \%)$ & $37(2.5 \%)$ & $52(3.6 \%)$ & $0(0.0 \%)$ \\
\hline Infectious diseases & $69(2.2 \%)$ & $23(1.5 \%)$ & $44(3.1 \%)$ & $2(0.7 \%)$ \\
\hline Intensive care & $86(2.7 \%)$ & $58(3.9 \%)$ & $24(1.7 \%)$ & $4(1.4 \%)$ \\
\hline Internal medicine & $1370(42.9 \%)$ & $665(44.5 \%)$ & $549(38.5 \%)$ & $156(56.3 \%)$ \\
\hline Laboratory medicine & $3(0.1 \%)$ & $0(0.0 \%)$ & $1(0.1 \%)$ & $2(0.7 \%)$ \\
\hline Obstetrics and gynecology & $133(4.2 \%)$ & $62(4.2 \%)$ & $58(4.1 \%)$ & $13(4.7 \%)$ \\
\hline Oncology & $74(2.3 \%)$ & $48(3.2 \%)$ & $21(1.5 \%)$ & $5(1.8 \%)$ \\
\hline Ophthalmology & $69(2.2 \%)$ & $21(1.4 \%)$ & $48(3.4 \%)$ & $0(0.0 \%)$ \\
\hline Orthopedic surgery, medical cosmetology & $11(0.3 \%)$ & $4(0.3 \%)$ & $7(0.5 \%)$ & $0(0.0 \%)$ \\
\hline Otolaryngology & $37(1.2 \%)$ & $12(0.8 \%)$ & $21(1.5 \%)$ & $4(1.4 \%)$ \\
\hline Pain medicine & $6(0.2 \%)$ & $0(0.0 \%)$ & $6(0.4 \%)$ & $0(0.0 \%)$ \\
\hline Pathology & $8(0.3 \%)$ & $3(0.2 \%)$ & $5(0.4 \%)$ & $0(0.0 \%)$ \\
\hline Pediatrics & $324(10.1 \%)$ & $138(9.2 \%)$ & $161(11.3 \%)$ & $25(9.0 \%)$ \\
\hline Psychiatry & $17(0.5 \%)$ & $14(0.9 \%)$ & $3(0.2 \%)$ & $0(0.0 \%)$ \\
\hline Radiology & $138(4.3 \%)$ & $69(4.6 \%)$ & $66(4.6 \%)$ & $3(1.1 \%)$ \\
\hline Sports medicine, rehabilitation & $86(2.7 \%)$ & $26(1.7 \%)$ & $49(3.4 \%)$ & $11(4.0 \%)$ \\
\hline Stomatology & $29(0.9 \%)$ & $5(0.3 \%)$ & $19(1.3 \%)$ & $5(1.8 \%)$ \\
\hline Surgery & 415 (13.0\%) & $245(16.4 \%)$ & $151(10.6 \%)$ & $19(6.9 \%)$ \\
\hline Traditional Chinese medicine & $39(1.2 \%)$ & $11(0.7 \%)$ & $25(1.8 \%)$ & $3(1.1 \%)$ \\
\hline Others & 104 (3.3\%) & 25 (1.7\%) & $58(4.1 \%)$ & $21(7.6 \%)$ \\
\hline
\end{tabular}

or treatment of a rare disease patient in their professional careers. Among the 3197 eligible participants in this study, over $60 \%$ of them obtained a total knowledge score of 2 or 3, which represented an average or good level of rare disease knowledge. Physicians' knowledge on rare diseases were generally investigated through self-reported questionnaires in previous studies $[4,15$, 17]. A study conducted in Belgium revealed that most of the investigated specialists scored their knowledge on rare diseases as average or good [17], while another international study indicated that the majority of specialists rated their current knowledge as excellent or good [4]. These prior studies focused on physicians' individual assessment of their knowledge on rare diseases in general and the responses can be varied depending on specialty, healthcare system, and the disease in physicians' mind when filling out the questionnaire [17]. Furthermore, previous studies developed questions to investigate physicians' education and information needs on rare diseases through expert interviews or previous publications [11, 17], however, these questionnaires may lack patient perspectives. To resolve the above uncertainties, the present study invited patient organizations with a unique disease of interest to develop questions so as to assess levels of disease-specific knowledge among physicians from an objective perspective. The findings in the current study can not only specify physicians' needs on rare disease knowledge precisely, but also provide insights into the improvement of quality of care for rare disease patients.

Among the 34 rare diseases of concern, the median knowledge score of 10 rare diseases were relatively low. Several factors may account for the low knowledge score of these rare diseases. Firstly, although the prevalence of rare diseases is generally low, the degree of rarity between different diseases still varies significantly, ranging from somewhat rare, rare, to extremely rare [6]. Due to the lack of medical encounters of extremely rare diseases, physicians may have insufficient knowledge on 
Table 2 Medians of the total knowledge score by the 34 rare diseases of concern

\begin{tabular}{|c|c|c|c|c|c|}
\hline Type of rare diseases & No. of physicians (\%) & $\begin{array}{l}\text { Total } \\
\text { Median (IQR) }\end{array}$ & Eastern & Central & Western \\
\hline Albinism & $466(14.58 \%)$ & $2.00(2.00)$ & $2.00(2.00)$ & $2.00(2.00)$ & $2.00(1.00)$ \\
\hline Hepatolenticular degeneration & $379(11.85 \%)$ & $2.00(1.00)$ & $2.00(1.00)$ & $2.00(1.00)$ & $2.00(1.00)$ \\
\hline Multiple sclerosis & $347(10.85 \%)$ & $1.00(1.00)$ & $1.00(1.00)$ & $1.00(0.00)$ & $1.00(0.00)$ \\
\hline Hemophilia & $293(9.16 \%)$ & $3.00(1.00)$ & $3.00(1.00)$ & $3.00(1.00)$ & $3.00(1.00)$ \\
\hline Marfan syndrome & $224(7.01 \%)$ & $2.00(0.00)$ & $2.00(0.00)$ & $2.00(0.00)$ & $2.00(0.00)$ \\
\hline Idiopathic pulmonary arterial hypertension & $168(5.25 \%)$ & $2.00(1.00)$ & $2.00(1.00)$ & $2.00(1.00)$ & $2.00(0.50)$ \\
\hline Systemic sclerosis & $152(4.75 \%)$ & $2.00(1.00)$ & $2.00(1.00)$ & $2.00(1.00)$ & $2.00(1.25)$ \\
\hline General myathenic gravis & $146(4.57 \%)$ & $2.00(2.00)$ & $2.00(1.00)$ & $1.50(1.75)$ & $2.00(2.00)$ \\
\hline Amyotrophic lateral sclerosis & $124(3.88 \%)$ & $1.00(1.00)$ & $1.00(1.00)$ & $1.00(1.00)$ & $2.00(0.00)$ \\
\hline Osteogenesis imperfecta & $118(3.69 \%)$ & $1.00(1.00)$ & $1.00(1.00)$ & $1.50(1.00)$ & $2.50(1.25)$ \\
\hline Langerhans cell histiocytosis & $110(3.44 \%)$ & $2.00(1.00)$ & $3.00(1.00)$ & $2.00(1.00)$ & $2.00(1.00)$ \\
\hline Neuromyelitis optica & $89(2.78 \%)$ & $2.00(0.00)$ & $2.00(0.00)$ & $2.00(0.00)$ & $2.00(0.50)$ \\
\hline Phenylketonuria & $71(2.22 \%)$ & $1.00(1.00)$ & $1.00(1.00)$ & $1.00(1.00)$ & $0.00(0.50)$ \\
\hline Duchenne muscular dystrophy & $67(2.10 \%)$ & $2.00(0.50)$ & $2.00(1.00)$ & $2.00(0.00)$ & $1.00(1.00)$ \\
\hline Lymphangioleiomyomatosis & $57(1.78 \%)$ & $2.00(1.00)$ & $2.00(1.00)$ & $2.00(0.25)$ & $2.00(0.00)$ \\
\hline Idiopathic hypogonadotropic hypogonadism & $49(1.53 \%)$ & $2.00(1.00)$ & $2.00(0.00)$ & $1.00(1.00)$ & $2.00(0.00)$ \\
\hline Prader-Willi syndrome & $46(1.44 \%)$ & $1.00(1.00)$ & $1.00(0.75)$ & $1.00(1.00)$ & $2.00(2.00)$ \\
\hline Congenital adrenal hypoplasia & $44(1.38 \%)$ & $2.00(1.00)$ & $2.00(1.00)$ & $2.00(1.00)$ & $2.00(0.00)$ \\
\hline Tuberous sclerosis complex & $38(1.19 \%)$ & $2.00(0.00)$ & $2.00(0.75)$ & $2.00(0.00)$ & $2.00(0.25)$ \\
\hline Spinal muscular atrophy & $30(0.94 \%)$ & $1.50(1.00)$ & $2.00(1.00)$ & $1.00(1.00)$ & $1.50(0.50)$ \\
\hline Kallmann syndrome & $26(0.81 \%)$ & $2.00(1.00)$ & $2.00(1.00)$ & $2.00(0.25)$ & $1.50(1.25)$ \\
\hline Homozygous hypercholesterolemia & $25(0.78 \%)$ & $2.00(1.00)$ & $2.00(0.75)$ & $1.00(1.00)$ & $2.00(0.50)$ \\
\hline Hereditary epidermolysis bullosa & $23(0.72 \%)$ & $2.00(1.00)$ & $2.00(1.00)$ & $1.00(1.00)$ & - \\
\hline Fabry disease & $20(0.63 \%)$ & $2.00(0.25)$ & $2.00(0.00)$ & $2.00(0.75)$ & $2.00(1.00)$ \\
\hline Huntington disease & $17(0.53 \%)$ & $2.00(1.00)$ & $2.00(1.00)$ & $2.00(1.00)$ & $1.00(0.50)$ \\
\hline Gaucher disease & $16(0.50 \%)$ & $2.00(1.25)$ & $2.00(1.00)$ & $3.00(0.75)$ & $2.00(0.00)$ \\
\hline Spinocerebellar ataxia & $15(0.47 \%)$ & $1.00(1.50)$ & $1.00(2.00)$ & $0.50(1.00)$ & $0.50(0.50)$ \\
\hline Niemann-Pick disease & $12(0.38 \%)$ & $3.00(1.00)$ & $3.00(1.00)$ & $3.00(1.00)$ & - \\
\hline Mucopolysaccharidosis & $6(0.19 \%)$ & $2.00(0.75)$ & $2.00(0.00)$ & $2.50(0.50)$ & $2.50(0.50)$ \\
\hline Severe myoclonic epilepsy in infaricy & $6(0.19 \%)$ & $2.00(1.50)$ & $2.00(0.00)$ & $1.00(1.00)$ & $2.00(1.00)$ \\
\hline Hyperphenylalaninemia & $5(0.16 \%)$ & $2.00(1.00)$ & $1.00(0.00)$ & $2.50(0.50)$ & $2.00(0.00)$ \\
\hline Spinal and bulbar muscular atrophy & $4(0.13 \%)$ & $1.00(0.25)$ & $0.50(0.50)$ & $1.00(0.00)$ & - \\
\hline Type II glycogen storage disease & $3(0.09 \%)$ & $1.00(0.50)$ & $1.00(0.00)$ & $1.50(0.50)$ & - \\
\hline Tetrahydrobiopterin deficiency & $1(0.03 \%)$ & $1.00(0.00)$ & $1.00(0.00)$ & - & - \\
\hline
\end{tabular}

-No physicians answered the relevant knowledge questions

these diseases such as Tetrahydrobiopterin deficiency with a prevalence rate of 1-9/1000,000 [18]. Secondly, technological advances on the diagnosis and treatment of rare diseases are rapidly evolving in recent years, however, physicians may not fully understand the translation process of the new technology into clinical practice, resulting in low correct rates of relevant questions, for instance, Amyotrophic lateral sclerosis Q2 and Multiple sclerosis Q2 (Additional file 2). Third, the majority of rare diseases are genetic in origin, many of which are caused by defects in a single gene or multiple genetic mutations [19]. However, physicians may receive lack of training and knowledge on genetics, resulting in a bad performance in answering inheritance-related questions such as Spinal muscular atrophy Q3, Prader-Willi syndrome Q3, and Spinal and bulbar muscular atrophy Q3 (Additional file 2). Fourth, due to lack of effective treatment for some rare diseases, patients with such diseases can only receive supportive treatment options including nutrition therapies, psychological treatment, and rehabilitation support. This situation often requires multidisciplinary expertise and communication, while insufficient 
Table 3 Characteristics of physicians with a total knowledge score ranging from a score of 0 to 3

\begin{tabular}{|c|c|c|c|c|c|}
\hline \multirow[t]{2}{*}{ Characteristics } & Score of 0 & Score of 1 & Score of 2 & Score of 3 & $p$-value \\
\hline & \multicolumn{5}{|c|}{ Number (percent) } \\
\hline Physician number & 224 (7.01\%) & 858 (26.84\%) & 1261 (39.44\%) & 854 (26.71\%) & \\
\hline Gender & & & & & $<0.001$ \\
\hline Male & $118(8.10 \%)$ & 417 (28.64\%) & $580(39.84 \%)$ & 341 (23.42\%) & \\
\hline Female & 106 (6.09\%) & 441 (25.33\%) & $681(39.12 \%)$ & $513(29.47 \%)$ & \\
\hline Age & & & & & 0.254 \\
\hline $18-25$ & $5(12.20 \%)$ & 10 (24.39\%) & $15(36.59 \%)$ & $11(26.83 \%)$ & \\
\hline $26-35$ & $93(6.53 \%)$ & $376(26.40 \%)$ & $554(38.90 \%)$ & 401 (28.16\%) & \\
\hline $36-45$ & 64 (5.95\%) & 302 (28.09\%) & $434(40.37 \%)$ & 275 (25.58\%) & \\
\hline $46-55$ & 47 (8.64\%) & 145 (26.65\%) & $214(39.34 \%)$ & 138 (25.37\%) & \\
\hline $56-65$ & $14(13.21 \%)$ & $24(22.64 \%)$ & $42(39.62 \%)$ & $26(24.53 \%)$ & \\
\hline $66+$ & $1(14.29 \%)$ & $1(14.29 \%)$ & $2(28.57 \%)$ & $3(42.86 \%)$ & \\
\hline Birthplace & & & & & 0.045 \\
\hline Urban area & $192(6.75 \%)$ & 774 (27.22\%) & 1133 (39.85\%) & 744 (26.17\%) & \\
\hline Rural area & $31(8.83 \%)$ & 84 (23.93\%) & $127(36.18 \%)$ & 109 (31.05\%) & \\
\hline Others & $1(33.33 \%)$ & $0(0.00 \%)$ & $1(33.33 \%)$ & $1(33.33 \%)$ & \\
\hline Monthly income & & & & & $<0.001$ \\
\hline$<5000$ & 70 (10.72\%) & 187 (28.64\%) & $241(36.91 \%)$ & 155 (23.74\%) & \\
\hline $5000-10,000$ & $133(6.56 \%)$ & 538 (26.55\%) & $783(38.65 \%)$ & $572(28.23 \%)$ & \\
\hline $10,000-30,000$ & $19(3.75 \%)$ & 131 (25.89\%) & $233(46.05 \%)$ & $123(24.31 \%)$ & \\
\hline $30,000-50,000$ & $2(28.57 \%)$ & $0(0.00 \%)$ & $2(28.57 \%)$ & $3(42.86 \%)$ & \\
\hline$>50,000$ & $0(0.00 \%)$ & $2(40.00 \%)$ & $2(40.00 \%)$ & $1(20.00 \%)$ & \\
\hline Province & & & & & $<0.001$ \\
\hline Jiangsu & $56(4.69 \%)$ & $303(25.36 \%)$ & $498(41.67 \%)$ & $338(28.28 \%)$ & \\
\hline Jiangxi & $39(8.33 \%)$ & $122(26.07 \%)$ & $172(36.75 \%)$ & $135(28.85 \%)$ & \\
\hline Shandong & $20(6.71 \%)$ & 99 (33.22\%) & $101(33.89 \%)$ & $78(26.17 \%)$ & \\
\hline Shanxi & 95 (9.91\%) & $259(27.01 \%)$ & $371(38.69 \%)$ & $234(24.40 \%)$ & \\
\hline Shannxi & $7(7.07 \%)$ & $29(29.29 \%)$ & $40(40.40 \%)$ & $23(23.23 \%)$ & \\
\hline Sichuan & $7(3.93 \%)$ & $46(25.84 \%)$ & $79(44.38 \%)$ & $46(25.84 \%)$ & \\
\hline Education & & & & & $<0.001$ \\
\hline Secondary vocational school & $2(66.67 \%)$ & $1(33.33 \%)$ & $0(0.00 \%)$ & $0(0.00 \%)$ & \\
\hline Three-year college & $2(10.53 \%)$ & $8(42.11 \%)$ & $7(36.84 \%)$ & $2(10.53 \%)$ & \\
\hline Bachelor's degree & $111(10.05 \%)$ & $327(29.62 \%)$ & $389(35.24 \%)$ & 277 (25.09\%) & \\
\hline Master's degree & $94(5.27 \%)$ & $449(25.20 \%)$ & $741(41.58 \%)$ & $498(27.95 \%)$ & \\
\hline Doctorate/postdoc & $15(5.19 \%)$ & $73(25.26 \%)$ & $124(42.91 \%)$ & $77(26.64 \%)$ & \\
\hline Title & & & & & 0.153 \\
\hline Primary & $69(8.38 \%)$ & $213(25.88 \%)$ & $302(36.70 \%)$ & $239(29.04 \%)$ & \\
\hline Middle & $72(5.96 \%)$ & 338 (27.98\%) & $500(41.39 \%)$ & 298 (24.67\%) & \\
\hline Vice-senior & $47(6.63 \%)$ & 194 (27.36\%) & $285(40.20 \%)$ & 183 (25.81\%) & \\
\hline Senior & $36(8.28 \%)$ & $108(24.83 \%)$ & $163(37.47 \%)$ & $128(29.43 \%)$ & \\
\hline None & $0(0.00 \%)$ & $5(22.73 \%)$ & $11(50.00 \%)$ & $6(27.27 \%)$ & \\
\hline Working years & & & & & 0.348 \\
\hline$\leq 5$ & $59(7.13 \%)$ & 203 (24.52\%) & $323(39.01 \%)$ & 243 (29.35\%) & \\
\hline $6-10$ & $41(5.26 \%)$ & $222(28.50 \%)$ & $316(40.56 \%)$ & $200(25.67 \%)$ & \\
\hline $11-15$ & $36(7.00 \%)$ & $146(28.40 \%)$ & $200(38.91 \%)$ & $132(25.68 \%)$ & \\
\hline $16-20$ & $26(7.07 \%)$ & 106 (28.80\%) & $144(39.13 \%)$ & 92 (25.00\%) & \\
\hline $21-25$ & $26(7.81 \%)$ & 87 (26.13\%) & $122(36.64 \%)$ & 98 (29.43\%) & \\
\hline $26-30$ & $17(7.87 \%)$ & $55(25.46 \%)$ & 91 (42.13\%) & $53(24.54 \%)$ & \\
\hline $30+$ & 19 (11.95\%) & $39(24.53 \%)$ & 65 (40.88\%) & $36(22.64 \%)$ & \\
\hline
\end{tabular}


Table 3 (continued)

\begin{tabular}{|c|c|c|c|c|c|}
\hline \multirow[t]{2}{*}{ Characteristics } & Score of 0 & Score of 1 & Score of 2 & Score of 3 & $p$-value \\
\hline & \multicolumn{5}{|c|}{ Number (percent) } \\
\hline Hospital class & & & & & 0.008 \\
\hline Primary hospital & $2(50.00 \%)$ & $1(25.00 \%)$ & $1(25.00 \%)$ & $0(0.00 \%)$ & \\
\hline Secondary hospital & $2(25.00 \%)$ & $4(50.00 \%)$ & $1(12.50 \%)$ & $1(12.50 \%)$ & \\
\hline Tertiary hospital & 220 (6.91\%) & $853(26.78 \%)$ & $1259(39.53 \%)$ & $853(26.78 \%)$ & \\
\hline Specialty & & & & & $<0.001$ \\
\hline Internal medicine & $59(4.31 \%)$ & $325(23.72 \%)$ & $563(41.09 \%)$ & $423(30.88 \%)$ & \\
\hline Anesthesiology & $3(9.09 \%)$ & $15(45.45 \%)$ & $12(36.36 \%)$ & $3(9.09 \%)$ & \\
\hline Dermatology & $0(0.00 \%)$ & $7(12.28 \%)$ & $31(54.39 \%)$ & $19(33.33 \%)$ & \\
\hline Emergency medicine & $6(6.74 \%)$ & $23(25.84 \%)$ & $40(44.94 \%)$ & $20(22.47 \%)$ & \\
\hline Infectious dept & $5(7.25 \%)$ & $9(13.04 \%)$ & $34(49.28 \%)$ & $21(30.43 \%)$ & \\
\hline Intensive care unit & $7(8.14 \%)$ & $29(33.72 \%)$ & $30(34.88 \%)$ & $20(23.26 \%)$ & \\
\hline Laboratory dept & $0(0.00 \%)$ & $1(33.33 \%)$ & $2(66.67 \%)$ & $0(0.00 \%)$ & \\
\hline Obstetrics and gynecology & $13(9.77 \%)$ & $40(30.08 \%)$ & $50(37.59 \%)$ & $30(22.56 \%)$ & \\
\hline Oncology & $6(8.11 \%)$ & $29(39.19 \%)$ & $20(27.03 \%)$ & $19(25.68 \%)$ & \\
\hline Ophthalmology & $2(2.90 \%)$ & $19(27.54 \%)$ & $28(40.58 \%)$ & $20(28.99 \%)$ & \\
\hline Orthopedic surgery, medical cosmetology & $3(27.27 \%)$ & $1(9.09 \%)$ & $3(27.27 \%)$ & $4(36.36 \%)$ & \\
\hline Otolaryngology & $6(16.22 \%)$ & $11(29.73 \%)$ & $12(32.43 \%)$ & $8(21.62 \%)$ & \\
\hline Pain medicine & $0(0.00 \%)$ & $0(0.00 \%)$ & $5(83.33 \%)$ & $1(16.67 \%)$ & \\
\hline Pathology & $0(0.00 \%)$ & $1(12.50 \%)$ & $7(87.50 \%)$ & $0(0.00 \%)$ & \\
\hline Pediatrics & $26(8.02 \%)$ & $69(21.30 \%)$ & $119(36.73 \%)$ & $110(33.95 \%)$ & \\
\hline Psychiatry & $2(11.76 \%)$ & $8(47.06 \%)$ & $4(23.53 \%)$ & $3(17.65 \%)$ & \\
\hline Radiology & $13(9.42 \%)$ & $46(33.33 \%)$ & $59(42.75 \%)$ & $20(14.49 \%)$ & \\
\hline Sports medicine, rehabilitation & $12(13.95 \%)$ & $29(33.72 \%)$ & $32(37.21 \%)$ & $13(15.12 \%)$ & \\
\hline Stomatology & $5(17.24 \%)$ & $8(27.59 \%)$ & $12(41.38 \%)$ & $4(13.79 \%)$ & \\
\hline Surgery & $41(9.88 \%)$ & 145 (34.94\%) & $149(35.90 \%)$ & $80(19.28 \%)$ & \\
\hline Traditional Chinese medicine & $5(12.82 \%)$ & $16(41.03 \%)$ & $12(30.77 \%)$ & $6(15.38 \%)$ & \\
\hline Others & 10 (9.62\%) & 27 (25.96\%) & 37 (35.58\%) & 30 (28.85\%) & \\
\hline
\end{tabular}

multidisciplinary collaboration may lead to specialists' limited knowledge on specific rare diseases [11, 15, 17], for example, Phenylketouria Q2 and Q3 (Additional file 2). In general, apart from identifying physicians' characteristics associated with the knowledge score on rare diseases, it's also important to investigate the relationship between physicians' knowledge on rare diseases and disease attributes (e.g., a disease with no treatment or with therapies in development) in future studies.

Factors associated with the knowledge score were identified in the multiple linear regression analysis. Females were more likely to obtain a higher knowledge score than males. Physicians with a monthly income of 5000-10,000 RMB and 10,000-30,000 RMB were associated with a higher knowledge score. This finding suggested that the socioeconomic status of physicians could affect their performance on rare disease knowledge. Hospitals locates in eastern China usually have access to more healthcare resources and insurance subsidies from the government. Hence, physicians from eastern China are more likely to receive a relatively higher income. Due to the uneven distribution of healthcare in China, patients have to travel across regions to access high-quality healthcare for the diagnosis and treatment of rare diseases [16]. Physicians from eastern China have more opportunities to encounter rare disease patients and thus increase related knowledge. On the contrary to our intuition, physicians working 5 years or below were more likely to obtain a higher knowledge score than physicians working longer than 30 years. This finding may indicate that working experience is not enough for accumulating rare disease knowledge. Medical school education and continuing training also play an important role to raise awareness of physicians on rare diseases; such education and training have gradually increased over time [15]. Several specialties of physicians including internal medicine, obstetrics and gynecology, radiology, intensive care unit, and surgery, had a comparably better performance in knowledge score after controlling for the other variables. These specialties of physicians had medical encounters of either a 
Table 4 Factors associated with the total knowledge score among physicians in China

\begin{tabular}{|c|c|c|}
\hline \multirow[t]{2}{*}{ Variables } & \multicolumn{2}{|l|}{ Coefficients $[95 \% \mathrm{Cl}]$} \\
\hline & Univariable & Multivariable \\
\hline \multicolumn{3}{|l|}{ Gender } \\
\hline Male & Reference & Reference \\
\hline Female & $0.13[0.07,0.20]^{* * *}$ & $0.08[0.01,0.14]^{*}$ \\
\hline \multicolumn{3}{|l|}{ Age } \\
\hline $18-25$ & Reference & \\
\hline $26-35$ & $0.11[-0.17,0.38]$ & \\
\hline $36-45$ & $0.08[-0.20,0.35]$ & \\
\hline $46-55$ & $0.03[-0.25,0.32]$ & \\
\hline $56-65$ & $-0.03[-0.35,0.30]$ & \\
\hline $66+$ & $0.22[-0.50,0.93]$ & - \\
\hline \multicolumn{3}{|l|}{ Birthplace } \\
\hline Urban area & Reference & - \\
\hline Rural area & $0.04[-0.06,0.14]$ & \\
\hline Others & $-0.19[-1.20,0.82]$ & \\
\hline \multicolumn{3}{|l|}{ Monthly income } \\
\hline$<5000$ & Reference & Reference \\
\hline $5000-10,000$ & $0.15[0.07,0.23]^{* * *}$ & $0.11[0.03,0.18]^{* *}$ \\
\hline $10,000-30,000$ & $0.17[0.07,0.28]^{* *}$ & $0.14[0.03,0.25]^{*}$ \\
\hline $30,000-50,000$ & $0.12[-0.54,0.78]$ & $-0.03[-0.62,0.56]$ \\
\hline$>50,000$ & $0.06[-0.72,0.85]$ & $0.21[-0.48,0.91]$ \\
\hline \multicolumn{3}{|l|}{ Province } \\
\hline Jiangsu & Reference & Reference \\
\hline Shandong & $-0.14[-0.25,-0.03]^{*}$ & $-0.10[-0.20,0.00]$ \\
\hline Jiangxi & $-0.07[-0.17,0.02]$ & $-0.04[-0.13,0.05]$ \\
\hline Shanxi & $-0.16[-0.24,-0.08]^{* * *}$ & $-0.10[-0.18,-0.03]^{* *}$ \\
\hline Sichuan & $-0.01[-0.15,0.13]$ & $0.02[-0.11,0.15]$ \\
\hline Shannxi & $-0.14[-0.32,0.04]$ & $-0.19[-0.36,-0.01]^{*}$ \\
\hline \multicolumn{3}{|l|}{ Education } \\
\hline Secondary vocational school & Reference & Reference \\
\hline Three-year college & $1.14[0.06,2.22]^{*}$ & $0.36[-0.64,1.37]$ \\
\hline Bachelor & $1.42[0.41,2.43]^{* *}$ & $0.53[-0.41,1.47]$ \\
\hline Master & $1.59[0.58,2.59]^{* *}$ & $0.63[-0.31,1.57]$ \\
\hline Doctor/postdoc & $1.58[0.57,2.59]^{* *}$ & $0.61[-0.33,1.56]$ \\
\hline \multicolumn{3}{|l|}{ Title } \\
\hline Primary & Reference & - \\
\hline Middle & $-0.02[-0.10,0.06]$ & \\
\hline Vice-senior & $-0.01[-0.10,0.08]$ & \\
\hline Senior & $0.02[-0.09,0.12]$ & \\
\hline None & $0.18[-0.20,0.56]$ & \\
\hline \multicolumn{3}{|l|}{ Working years } \\
\hline$\leq 5$ & Reference & Reference \\
\hline $6-10$ & $-0.04[-0.13,0.05]$ & $-0.05[-0.13,0.03]$ \\
\hline $11-15$ & $-0.07[-0.17,0.03]$ & $0.00[-0.10,0.09]$ \\
\hline $16-20$ & $-0.09[-0.19,0.02]$ & $-0.05[-0.16,0.05]$ \\
\hline $21-25$ & $-0.03[-0.14,0.08]$ & $0.01[-0.09,0.12]$ \\
\hline $26-30$ & $-0.07[-0.21,0.06]$ & $-0.04[-0.17,0.09]$ \\
\hline $30+$ & $-0.16[-0.32,-0.01]^{*}$ & $-0.18[-0.32,-0.03]^{*}$ \\
\hline
\end{tabular}


Table 4 (continued)

\begin{tabular}{|c|c|c|}
\hline \multirow[t]{2}{*}{ Variables } & \multicolumn{2}{|l|}{ Coefficients $[95 \% \mathrm{Cl}]$} \\
\hline & Univariable & Multivariable \\
\hline \multicolumn{3}{|l|}{ Hospital class } \\
\hline Primary hospital & Reference & Reference \\
\hline Secondary hospital & $0.38[-0.69,1.44]$ & $0.19[-0.79,1.17]$ \\
\hline Tertiary hospital & $1.11[0.24,1.99]^{*}$ & $0.33[-0.48,1.15]$ \\
\hline \multicolumn{3}{|l|}{ Specialty } \\
\hline Internal medicine & Reference & Reference \\
\hline Anesthesiology & $-0.53[-0.83,-0.23]^{* *}$ & $-0.45[-0.72,-0.17]^{* *}$ \\
\hline Dermatology & $0.23[-0.01,0.46]$ & $0.18[-0.04,0.40]$ \\
\hline Emergency medicine & $-0.15[-0.34,0.03]$ & $-0.11[-0.29,0.06]$ \\
\hline Infectious disease & $0.04[-0.17,0.26]$ & $-0.06[-0.26,0.14]$ \\
\hline Intensive care unit & $-0.25[-0.44,-0.06]^{*}$ & $-0.20[-0.37,-0.02]^{*}$ \\
\hline Laboratory department & $-0.32[-1.31,0.68]$ & $-0.13[-1.03,0.77]$ \\
\hline Obstetrics and gynecology & $-0.26[-0.41,-0.10]^{* *}$ & $-0.15[-0.30,-0.01]^{*}$ \\
\hline Oncology & $-0.28[-0.49,-0.08]^{* *}$ & $-0.38[-0.56,-0.19]^{* * *}$ \\
\hline Ophthalmology & $-0.03[-0.24,0.18]$ & $0.00[-0.20,0.20]$ \\
\hline Orthopedic surgery, medical cosmetology & $-0.26[-0.78,0.26]$ & $-0.20[-0.66,0.27]$ \\
\hline Otolaryngology & $-0.39[-0.68,-0.10]^{* *}$ & $-0.46[-0.72,-0.20]^{* *}$ \\
\hline Pain medicine & $0.18[-0.52,0.89]$ & $0.36[-0.27,0.99]$ \\
\hline Pathology & $-0.11[-0.72,0.50]$ & $-0.44[-1.00,0.11]$ \\
\hline Pediatrics & $-0.02[-0.13,0.09]$ & $0.08[-0.02,0.19]$ \\
\hline Psychiatry & $-0.51[-0.93,-0.09]^{*}$ & $-0.43[-0.81,-0.05]^{*}$ \\
\hline Radiology & $-0.36[-0.52,-0.21]^{* * *}$ & $-0.16[-0.30,-0.02]^{*}$ \\
\hline Sports medicine, rehabilitation & $-0.45[-0.64,-0.26]^{* * *}$ & $-0.16[-0.33,0.02]$ \\
\hline Stomatology & $-0.47[-0.79,-0.15]^{* *}$ & $-0.63[-0.93,-0.34]^{* * *}$ \\
\hline Surgery & $-0.34[-0.44,-0.24]^{* * *}$ & $-0.24[-0.34,-0.14]^{* * *}$ \\
\hline Traditional Chinese medicine & $-0.50[-0.78,-0.22]^{* * *}$ & $-0.19[-0.45,0.06]$ \\
\hline Others & $-0.15[-0.32,0.03]$ & $-0.06[-0.22,0.10]$ \\
\hline \multicolumn{3}{|l|}{ Rare disease type } \\
\hline Albinism & Reference & Reference \\
\hline Osteogenesis imperfecta & $-0.29[-0.45,-0.13]^{* * *}$ & $-0.28[-0.44,-0.12]^{* *}$ \\
\hline Homozygous hypercholesterolemia & $-0.27[-0.59,0.05]$ & $-0.42[-0.74,-0.10]^{*}$ \\
\hline Duchenne muscular dystrophy & $0.11[-0.10,0.31]$ & $-0.09[-0.30,0.12]$ \\
\hline Multiple sclerosis & $-0.53[-0.64,-0.42]^{* * *}$ & $-0.64[-0.75,-0.52]^{* * *}$ \\
\hline Fabry disease & $0.26[-0.09,0.62]$ & $0.07[-0.28,0.42]$ \\
\hline Hepatolenticular degeneration & $0.38[0.27,0.48]^{* * *}$ & $0.25[0.13,0.36]^{* * *}$ \\
\hline Gaucher disease & $0.15[-0.25,0.55]$ & $-0.04[-0.43,0.36]$ \\
\hline Huntington disease & $-0.02[-0.41,0.36]$ & $-0.03[-0.41,0.35]$ \\
\hline Amyotrophic lateral sclerosis & $-0.22[-0.38,-0.07]^{* *}$ & $-0.33[-0.49,-0.17]^{* * *}$ \\
\hline Spinocerebellar ataxia & $-0.65[-1.06,-0.24]^{* *}$ & $-0.69[-1.10,-0.29]^{* *}$ \\
\hline Spinal muscular atrophy & $-0.39[-0.68,-0.09]^{*}$ & $-0.50[-0.80,-0.21]^{* *}$ \\
\hline Spinal and bulbar muscular atrophy & $-1.04[-1.82,-0.25]^{* *}$ & $-1.00[-1.78,-0.23]^{*}$ \\
\hline Tuberous sclerosis complex & $0.03[-0.24,0.29]$ & $-0.10[-0.37,0.16]$ \\
\hline Kallmann syndrome & $0.02[-0.29,0.34]$ & $-0.14[-0.46,0.17]$ \\
\hline Langerhans cell histiocytosis & $0.60[0.44,0.77]^{* * *}$ & $0.55[0.38,0.72]^{* * *}$ \\
\hline Lymphangioleiomyomatosis & $-0.12[-0.34,0.10]$ & $-0.28[-0.50,-0.06]^{*}$ \\
\hline Marfan syndrome & $0.07[-0.06,0.19]$ & $0.00[-0.13,0.12]$ \\
\hline Niemann-Pick disease & $0.71[0.26,1.17]^{* *}$ & $0.64[0.18,1.09]^{* *}$ \\
\hline Mucopolysaccharidosis & $0.55[-0.10,1.19]$ & $0.31[-0.32,0.95]$ \\
\hline
\end{tabular}


Table 4 (continued)

\begin{tabular}{lll}
\hline Variables & Coefficients $[95 \% \mathbf{C l}]$ & Multivariable \\
\cline { 2 - 3 } & Univariable & $-0.79[-1.04,-0.55]^{* * *}$ \\
\hline Prader-Willi syndrome & $-0.53[-0.77,-0.29]^{* * *}$ & $0.05[-0.10,0.20]$ \\
General myathenic gravis & $0.08[-0.07,0.22]$ & $0.03[-0.16,0.21]$ \\
Neuromyelitis optica & $0.20[0.02,0.38]^{*}$ & $-0.66[-1.55,0.23]$ \\
Type Il glycogen storage disease & $-0.45[-1.36,0.45]$ & $-0.28[-0.52,-0.05]^{*}$ \\
Idiopathic hypogonadotropic hypogonadism & $-0.09[-0.33,0.14]$ & $0.33[0.19,0.48]^{* * *}$ \\
Idiopathic pulmonary arterial hypertension & $0.48[0.34,0.62]^{* * *}$ & $0.32[0.17,0.47]^{* * *}$ \\
Systemic sclerosis & $0.50[0.36,0.65]^{* * *}$ & $0.01[-0.23,0.26]$ \\
Congenital adrenal hypoplasia & $0.24[-0.01,0.48]$ & $0.58[0.46,0.69]^{* * *}$ \\
Hemophilia & $0.64[0.52,0.76]^{* * *}$ & $-0.52[-0.85,-0.19]^{* *}$ \\
Hereditary epidermolysis bullosa & $-0.35[-0.69,-0.02]^{*}$ & $-0.54[-1.18,0.09]$ \\
Severe myoclonic epilepsy in infaricy & $-0.45[-1.10,0.19]$ & $-0.19[-0.88,0.50]$ \\
Hyperphenylalaninemia & $0.01[-0.69,0.72]$ & $-1.18[-1.38,-0.97]^{* * *}$ \\
Phenylketonuria & $-1.13[-1.32,-0.93]^{* * *}$ & $-1.05[-2.59,0.48]$ \\
Tetrahydrobiopterin deficiency & $-0.79[-2.35,0.78]$ & \\
\hline
\end{tabular}

${ }^{*} p<0.05 ;{ }^{* *} p<0.01 ;{ }^{* * *} p<0.001$

- Not included in the multiple linear regression analysis

large number of rare disease patients or a great variety of rare diseases, which contributed to the accumulation of rare disease knowledge (Additional file 3).

Although rare diseases only affect a small proportion of the general population, some specialties of physicians may encounter a large number of rare disease patients or a great variety of rare diseases in China. These specialties of physicians may have better performance on specific rare diseases, however, it's unrealistic for them to be experts for all the rare diseases that they encounter during their professional lives. This situation is not unique to China, but also common in different healthcare systems around the world [20]. Under this scenario, physicians should support the patient participation in medical encounters by sharing knowledge openly and encouraging patients' self-reliance. Additionally, patient organizations can play a critical role in providing rare disease patients with services and resources that are not otherwise available through expert patients or invited physicians $[15,21,22]$. Apart from the positive sustainment of patients' information-seeking process, it is equally important to enhance the pooling of knowledge on rare diseases by the implementation of multidisciplinary and digital platform, and to establish an effective referral method at the healthcare system level. Due to the low prevalence and scattered population of rare disease patients in China, tertiary hospitals located in big cities could establish a regular board of advisors by including experts from various specialties to further improve the diagnosis and treatment of rare diseases. An ideal digital platform was described as submitting patients' symptoms and test results and obtaining a differential diagnosis of rare diseases as output $[15,17]$. In China, despite the guideline for the diagnosis and treatment of rare diseases issued in 2019, more detailed information on differential diagnosis of rare diseases, referral services provided by experts or hospitals, and patient organizations are needed to guide medical services for rare diseases. Furthermore, Zhou and Nunes revealed that the key barrier to knowledge sharing in the provision of healthcare referral services in China is lack of leadership of the governance agencies in the process of inter-institutional knowledge sharing at the national and regional levels [23]. These problems need to be addressed to further enhance the implementation of knowledge sharing in the field of rare diseases in China.

To the best knowledge of us, this is the first national study investigating physicians' knowledge on 34 specific rare diseases in China. The study sample were recruited across eastern, central, and western China, which has increased the representativeness of the research findings. However, there are several limitations to this study. First, the knowledge questions developed in this study have not been validated, resulting in discrepancies in the difficulties of knowledge questions between different rare diseases. Future studies are needed to validate the difficulties of disease-specific knowledge questions so as to make the performance of physicians more comparable. Second, only 3 knowledge questions were used to measure the level of knowledge on a specific rare disease among physicians, lowering the reliability of the study findings. A more comprehensive questionnaire is 
required to assess the level of physicians' knowledge on specific rare diseases in future studies. Additionally, physicians were required to select only one rare disease that they felt most familiar with so as to compare their performance on disease-specific knowledge. However, physicians may encounter various types of rare diseases during their professional careers. Apart from their most familiar rare disease, future studies need to investigate physicians' knowledge on other specific rare diseases that they have encountered during their professional lives. Third, due to the selection bias of the sampling method, most of the participants in this study were specialists from tertiary hospitals in China. Several studies have indicated that the general practitioners' self-reported knowledge on rare diseases was worse than that of specialists $[11,17]$. Hence, the findings reported in the current study are not generalizable to a broader physician population in China. Fourth, selection bias may arise since the physicians included in the study reported their experience with rare diseases by themselves. Physicians that either declared having no medical encounters of rare disease patients or chose other rare diseases as their most familiar one beyond the specific 34 rare diseases of interest were not taken into account according to physicians' responses in this study. Although these physicians were excluded from the current study, it's still important to assess their knowledge on specific rare diseases using more objective data in future studies.

\section{Conclusions}

Almost two thirds of the participants had an average or good level of knowledge on the specific rare disease that they felt most experienced in. Physicians with female gender, a monthly income of 5000-10,000 RMB and 10,000-30,000 RMB, and specialties of internal medicine, obstetrics and gynecology, radiology, intensive care unit, and surgery, were associated with a relatively higher knowledge score. Medical education and continuous training programs, especially on genetics and translational research, are further needed to increase physicians' knowledge on rare diseases. Also, there are urgent needs of high-quality clinical guidelines and multidisciplinary collaboration to provide guidance on medical services for rare diseases in the healthcare system of China.

\section{Supplementary Information}

The online version contains supplementary material available at https://doi. org/10.1186/s13023-022-02243-7.

Additional file 1. List of knowledge questions and answers based on 34 rare diseases of concern.
Additional file 2. Correct rates of answering each of the three knowledge questions among physicians based on rare diseases.

Additional file 3. Frequencies of physicians in different specialties based on 34 rare diseases of concern.

\section{Acknowledgements}

We are grateful to all the 34 patient organizations for their great contributions to the development of knowledge questions in the process of study design.

\section{Authors' contributions}

ZSY and DD developed the idea; ZHY, XY, DD and ZSY designed the study; DD, $X Y, T Z$ and $Z X Y$ were responsible for data collection. $Z H Y$ and $X Y$ performed the statistical analysis and drafted the manuscript. ZXY, TZ, ZSY and DD revised it. All authors read and approved the final manuscript.

\section{Funding}

The study was funded by the '13th Five-Year' National Science and Technology Major Project for New Drugs (No: 2019ZX09734001), National Key Research and Development Program of China (2016YFC0901500), Beijing Natural Science Foundation (7192155), and Chinese Academy of Medical Sciences Innovation Fund for Medical Sciences (2021-I2M-1-003).

\section{Availability of data and materials}

All data generated or analyzed during this study are included in this published article and its Additional files.

\section{Declarations}

Ethics approval and consent to participate

This study was approved by the Peking Union Medical College Hospital Ethics Committee (Ref No.: SK-814). All the participants provided informed consents before the survey was conducted.

\section{Consent for publication}

Not applicable.

\section{Competing interests}

The authors declare that they have no competing interests.

\section{Author details}

'Shenzhen Research Institute, The Chinese University of Hong Kong, Shenzhen 518000, China. ${ }^{2}$ Department of Cardiology, Peking Union Medical College Hospital, Chinese Academy of Medical Sciences and Peking Union Medical College, Beijing 100730, China. ${ }^{3}$ JC School of Public Health and Primary Care, Faculty of Medicine, The Chinese University of Hong Kong, Shatin, Hong Kong SAR, China.

Received: 30 August 2021 Accepted: 10 February 2022

Published online: 05 March 2022

\section{References}

1. Richter T, Nestler-Parr S, Babela R, et al. Rare disease terminology and definitions-a systematic global review: report of the ISPOR rare disease special interest group. Value Health. 2015;18:906-14.

2. Schieppati A, Henter J-I, Daina E, Aperia A. Why rare diseases are an important medical and social issue. Lancet. 2008;371:2039-41.

3. Dawkins HJ, Draghia-Akli R, Lasko P, Lau LP, Jonker AH, Cutillo CM, Rath A, Boycott KM, Baynam G, Lochmüller H. Progress in rare diseases research 2010-2016: an IRDiRC perspective. Clin Transl Sci. 2018;11:11.

4. Engel PA, Bagal S, Broback M, Boice N. Physician and patient perceptions regarding physician training in rare diseases: the need for stronger educational initiatives for physicians. J Rare Disord. 2013;1 (2):1-15.

5. Domaradzki J, Walkowiak D. Medical students'knowledge and opinions about rare diseases: a case study from Poland. Intractable Rare Dis Res. 2019;8(4):252-9. 
6. Yan X, He S, Dong D. Determining how far an adult rare disease patient needs to travel for a definitive diagnosis: a cross-sectional examination of the 2018 national rare disease survey in China. Int J Environ Res Public Health. 2020;17(5):1757.

7. ISEBOX Ltd/Shire. Rare disease impact report. http://www.rarediseas eimpact.com. Published April 2013. Accessed 8 May 2021.

8. Eurordis. The voice of 12000 patients. Experiences and expectations of rare disease patients on diagnosis and care in Europe. 2009. https://www. eurordis.org/IMG/pdf/voice_12000_patients/EURORDISCARE_FULLB OOKr.pdf. Accessed 8 May 2021.

9. Budych K, Helms TM, Schultz C. How do patients with rare diseases experience the medical encounter? Health Policy. 2012;105:154-64.

10. Miteva T, Jordanova R, Iskrov G, Stefanov R. General knowledge and awareness on rare diseases among general practitioners in Bulgaria. Georg Med News. 2011;193:16-9.

11. Ramalle-Gómara E, Domínguez-Garrido E, Gómez-Eguílaz M, MarzoSola ME, Ramón-Trapero JL, Gil-De-Gómez J. Education and information needs for physicians about rare diseases in Spain. Orphanet J Rare Dis. 2020;15(1):1-7.

12. Huete A, Díaz E. Estudio sobre situación de necesidades Sociosanitarias de las personas con Enfermedades Raras en España. Estudio ENSERio. http://enfermedades-raras.org/images/stories/documentos/Estudio_ ENSERio.pdf.

13. Chiu ATG, Chung CCY, Wong WHS, Lee SL, Chung BHY. Healthcare burden of rare diseases in Hong Kong - adopting ORPHAcodes in ICD-10 based healthcare administrative datasets Dr. Segolene Ayme. Orphanet J Rare Dis. 2018;13(1):1-8.

14. Han J, Cui Y, Zhou X. Rare diseases research in China: opportunities, challenges, and solutions. Intract Rare Dis Res. 2012;1:10-2.

15. Li X, Zhang X, Zhang S, Lu Z, Zhang J, Zhou J, et al. Rare disease awareness and perspectives of physicians in China: a questionnaire-based study. Orphanet J Rare Dis. 2021;16(1):1-9.

16. Yan X, Dong D, He S, Webster C. Examining trans-provincial diagnosis of rare diseases in China: the importance of healthcare resource distribution and patient mobility. Sustainability. 2020;12(13):5444.

17. Vandeborne L, Van Overbeeke E, Dooms M, De Beleyr B, Huys I. Information needs of physicians regarding the diagnosis of rare diseases: a questionnaire-based study in Belgium. Orphanet J Rare Dis. 2019;14(1):1-11.

18. Orphanet. Hyperphenylalaninemia due to tetrahydrobiopterin deficiency. https://www.orpha.net/consor/cgi-bin/Disease.php?lng=EN [Cited 29 July 2021].

19. Institute of Medicine (US) Committee on Accelerating Rare Diseases Research and Orphan Product Development; Field MJ, Boat TF, editors Rare Diseases and Orphan Products: Accelerating Research and Development. Washington (DC): National Academies Press (US); 2010. 2, Profile of Rare Diseases. Available from: https://www.ncbi.nlm.nih.gov/books/ NBK56184/\#

20. Evans WR. Dare to think rare: diagnostic delay and rare diseases. Br J Gen Pract. 2018;68(670):224-5.

21. Gong S, Li D, Dong D. How do patients and doctors perceive medical services for rare diseases differently in China? Insights from two national surveys. Int J Environ Res Public Health. 2020;17(16):1-14.

22. Li X, Lu Z, Zhang J, Zhang X, Zhang S, Zhou J, et al. The urgent need to empower rare disease organizations in China: an interview-based study. Orphanet J Rare Dis. 2020;15(1):1-9.

23. Zhou L, Nunes MB. Barriers to knowledge sharing in Chinese healthcare referral services: an emergent theoretical model. Glob Health Action. 2016;9(1):29964.

\section{Publisher's Note}

Springer Nature remains neutral with regard to jurisdictional claims in published maps and institutional affiliations.
Ready to submit your research? Choose BMC and benefit from:

- fast, convenient online submission

- thorough peer review by experienced researchers in your field

- rapid publication on acceptance

- support for research data, including large and complex data types

- gold Open Access which fosters wider collaboration and increased citations

- maximum visibility for your research: over $100 \mathrm{M}$ website views per year

At BMC, research is always in progress.

Learn more biomedcentral.com/submissions 